\title{
Multi-Body Dynamics Simulation of A Tracked Vehicle Power Train in Consideration of Multi-Source Excitations
}

\author{
Hui $\mathrm{Li}^{1}$, Shengping $\mathrm{Fu}^{1,2}$, Changle Xiang ${ }^{1}$ \\ 1. National Key Laboratory of Vehicular Transmission, Beijing Institute of Technology, Beijing, P.R. CHINA \\ 2. State Key Laboratory of Advanced Design and Manufacture for Vehicle Body, College of Mechanical and Automotive \\ Engineering, Hunan University, Changsha, Hunan, P.R.CHINA
}

Received: 24-02-2011

Accepted: 20-04-2011

\begin{abstract}
This study proposed a new efficient method to investigate tracked vehicle gear transmission system dynamics applying Finite element theory and multi-body dynamics. Torsional excitation was simulated based on an engine dynamic model by considering flexibility of crankshaft, maximum fire pressure and variable inertia. Gear mesh stiffness coefficient, mesh error excitation, bearing dynamic stiffness coefficient and damping coefficient were numerically calculated. The virtual prototype model of power train system including all aforementioned excitations was constructed. Dynamic responses of transmission shaft working with multi-source excitations were analyzed. The impact of each excitation source was discussed.
\end{abstract}

Keywords: Multi-source dynamic excitations; Tracked vehicle; Power gear transmission system; Multi-body dynamic simulation

\section{Introduction}

The power train exhibits a wide range of dynamics behaviors due to multi-source variable excitations. The gear system dynamics has been extensively investigated. A.Kahraman ${ }^{1}$ studied the inner excitations of straight helical gear pair. The difference between outer and inner excitations was deeply analyzed. T.Tsuta ${ }^{2}$ viewed the result of gear meshing stiffness multiplying meshing error as the inner excitation. And one method of calculating meshing impact force was presented. R.G.Parker ${ }^{3}$ calculated the gear inner excitations including meshing stiffness and error and obtained the dynamic responses in different condition applying the finite element method. L.Vedmar ${ }^{4}$ analyzed the load characteristics of gear transmission system with the consideration of meshing stiffness, meshing dampness, teeth surface friction and bearing support stiffness. Li Runfang, Lin Tengjiao etc. ${ }^{5,6}$ obtained the time-dependent meshing stiffness, error excitation and meshing impact excitation by applying numerical

*Corresponding author: 7025370@163.com simulation. Excitation sources were brought to the finite element model of gear system to calculate the vibration responses. Yang Chengyun ${ }^{7}$ established the coupled vibration model of gear system including gear transmission system and structure system. The coupled vibration characteristic of gear system under the dynamic inner excitations was analyzed and the non-linear contact dynamic characteristic of gear transmission system was also studied. The research objects of these studies were seldom about tracked vehicle power-train. The outer excitations such as power source and support component were neglected. To efficiently deal with modeling of complex nonlinear dynamical systems, efficient computational models were required ${ }^{8}$. The finite element method was adopted to calculate most in these studies. Hence, the calculation efficiency was low and this method was not appropriate for the complicated mechanism.

Dynamics characteristics of power multistage gear transmission system have been analyzed by ADAMS ${ }^{9,12}$. Engine pure outer torque was equivalently brought to function in the prototype input terminal. Average gear mesh stiffness coefficient, fixed bearing support stiffness 
and dampness matrixes were used to compute system dynamics equation. Because of being beyond consideration of the time-varying characteristics of engine output torque, gear pair dynamic mesh traits and bearing fluctuant support parameters, the prototype in the papers was not in good agreement with real working condition of gear system. It is desired to construct the prototype of power gear train system with multi-source dynamic excitations to more precisely simulate the dynamics.

As shown in Fig. 1, this study considers multi-stage power gear train system, the main component of integrated transmission mechanism used in a certain tracked vehicle, with three freedoms. Engine dynamic output torque is obtained based on its prototype including flexible crankshaft by ADAMS. Time-dependent mesh characteristics of gear pair is studied by the contact finite element method. Dynamic contact traits of bearing are analyzed using the EHL lubrication condition and Hertz contact theory. After the crucial parts are described mathematically, the rigid and flexible coupled prototype of power gear transmission system is constructed by considering the effects of multi-source excitations. Steady system working at a given gear ratio is simulated for the engine at $2000 \mathrm{r} / \mathrm{min}$.

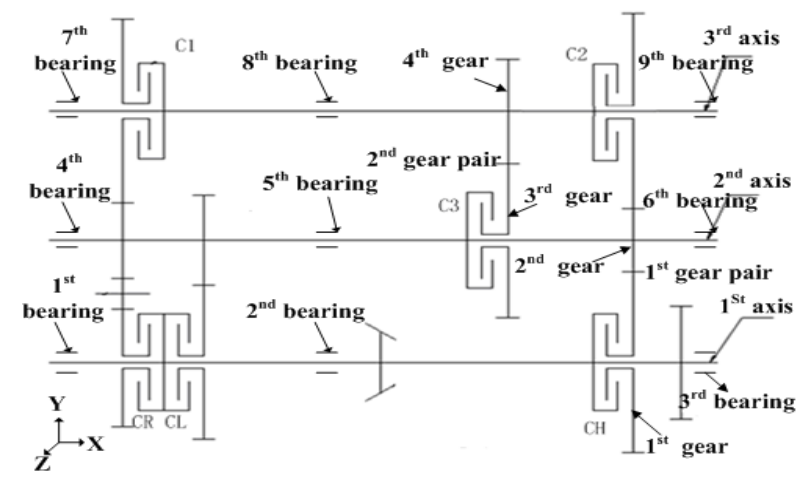

Fig.1. Schematic diagram of the transmission system

\section{Engine excitation}

\subsection{Engine dynamic model}

Engine dynamic excitation is modeled as the fluctuant output torque of engine crankshaft. If engine has equal ignition interval, its output torque is considered by adding the torque of each individual cylinder with a phase difference. Inner friction forces and outer resistances are ignored in this model. The abrasion and deformation of pistons and cylinders sleeves are also neglected. Crankshaft output torque is:

$$
T=T_{0}+\sum T^{\prime}
$$

where $T_{0}$ is the engine average output torque; $\sum T$ is the torsional excitation torque of crankshaft mechanism that includes the influence factor of maximum fire pressure, reciprocating parts variable inertia and gravity.

Type 4 stroke engine with 8 cylinders matches the research power gear train system. Mode information of crankshaft is obtained by ANSYS to consider the influence of crankshaft flexibility. The flexibility is imported into system dynamics equation by coordinate transformation. As shown in Fig. 2, if kinetic correlation of each part is selected, the engine dynamic model is established.

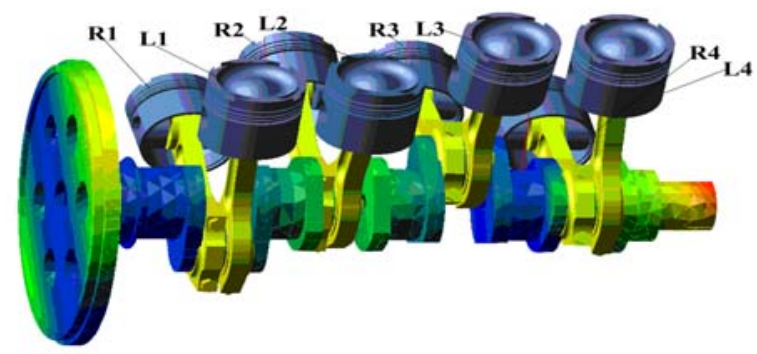

Fig.2. Engine dynamic model

The construction of engine dynamic model is based on a 3D solid model. With the mass and inertia of each part, numerical simulation in ADAMS takes the influence factor of variable inertia and gravity into consideration automatically.

Series of maximum fire pressures acting on the top piston are the group of forces fluctuating with the same dynamics except that the phases are different. As shown in Fig. 3, according to AKISPL interpolation function, combustion pressure of each cylinder is calculated based on indicator diagram shown Fig. 3 as the ignition order.

$$
F_{j}=\text { AKISPL(angle, 0, spline_shigongtu, 0). } \pi \cdot \mathrm{D}^{2} / 4 \text {. (2) }
$$

Where $F_{j}$ is the maximum fire pressure of cylinder $\mathrm{j}$; angle is the phase angle; spline_shigongtu is the spline curve; and $D$ is the cylinder diameter. 


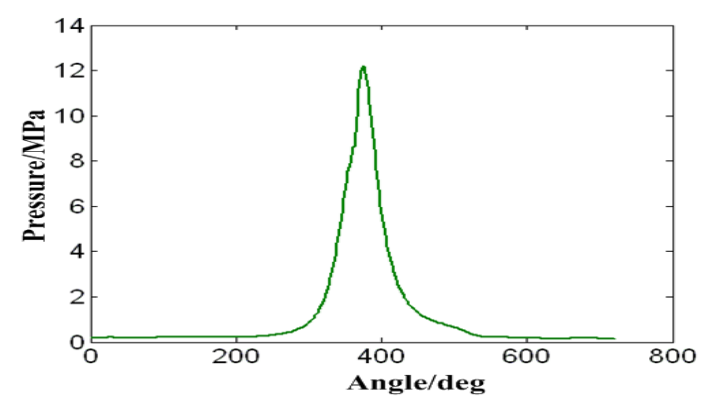

Fig.3. Eruption pressure of engine

\subsection{Simplifications of slide bearings}

Because of oil film flexibility in slide bearing, the connection between main crankshaft journal and bearing housing are flexible when engine works. The system is simplified into two spring joints with the same parameters in two radial directions. Bushing force is used to demonstrate contact trait of slide bearing as included in Fig. 4. The recommended values of stiffness and dampness in a diesel engine handbook are adopted.

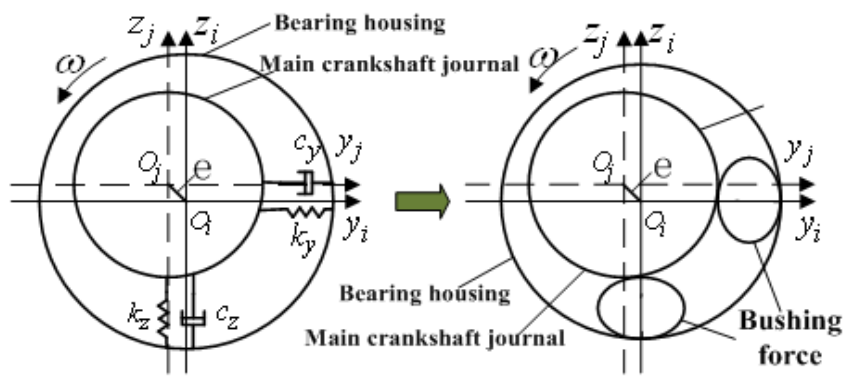

Fig.4. Schematic diagram of flexible support

The mathematic model is

$$
\left\{\begin{array}{l}
m \ddot{y}_{j}(t)+c_{y} \dot{y}_{j}(t)+k_{y}\left[y_{j}(t)-y_{i}(t)\right]=m \omega^{2} e \cos \omega t \\
m \ddot{z}_{j}(t)+c_{z} \dot{z}_{j}(t)+k_{z}\left[z_{j}(t)-z_{i}(t)\right]=m \omega^{2} e \sin \omega t
\end{array} .\right.
$$

where $O_{i}$ is the center of bearing housing; $O_{j}$ is the center of main crankshaft journal; $m$ is the mass of crankshaft; $c_{y}$ and $c_{z}$ are the radial support damping

coefficient; $k_{y}$ and $k_{z}$ are the radial support stiffness coefficients; $e$ is the eccentricity of shaft journal relative to the center of bearing; $\omega$ is the angular velocity of the crankshaft.

\subsection{Simulation results}

Engine works at 2000r/min steadily. Simulation time step is 0.001s. Each connecting rod journal load shown in Fig. 5 and crankshaft output torque shown in Fig. 6 are numerically acquired.



Fig.5. Connecting rod journal load

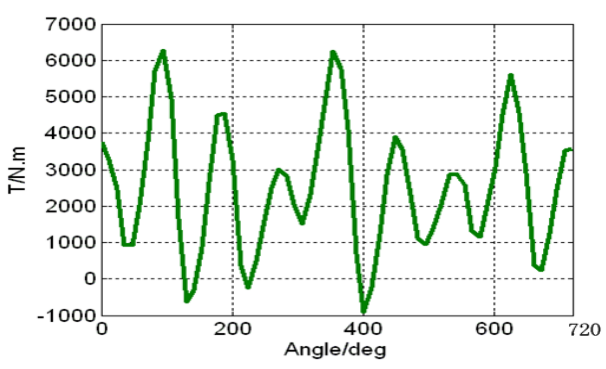

Fig.6. Output torque

\section{Gear inner excitation}

Mesh inner dynamic excitation is the primary cause of vibration and chaos in the gear box ${ }^{13}$. Mesh wallop resulted from the departure of meshing point is relatively small. Gear surface friction caused by lubrication is neglected in this study. Therefore, mesh stiffness coefficient performing as parameter excitation and mesh errors transformed to displacement excitation are studied to investigate the dynamic trait of power gear train system.

Mesh stiffness coefficient $k$ is the compositive value of $k_{i}$ which represents the stiffness of single couple of mesh gear teeth. It is equivalent to the value of load which makes several couples of gear teeth meshing at one 
time induce $1 \mu \mathrm{m}$ deformation of $1 \mathrm{~mm}$ tooth width ${ }^{13}$. It can be expressed as

$$
k=\sum_{i=1}^{n} k_{i}=\sum_{i=1}^{n} F_{i} /\left(\delta_{p i}+\delta_{g i}\right) .
$$

where $F_{i}$ is the mesh force; $\delta_{p i}$ and $\delta_{g i}$ are the tooth deformation of driving gear and driven gear separately; $n$ is the number of mesh teeth.

Mesh stiffness coefficient is calculated by assuming the gear tooth as a cantilever beam in this study. Simplified

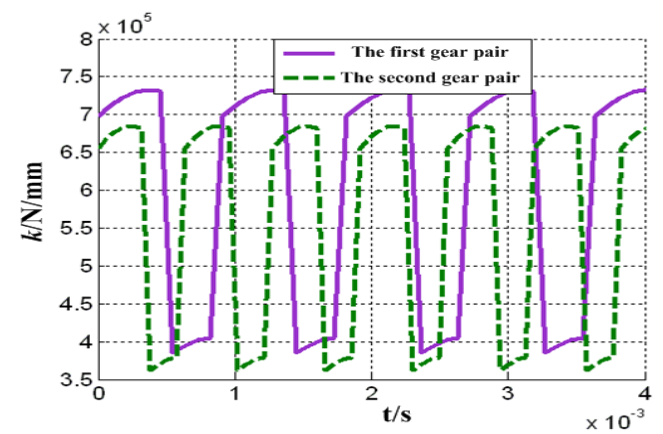

Fig.7. Mesh stiffness coefficient formulas were used to calculate the tooth deformation, which however is not in good agreement with real working condition. Hence, 3D contact dynamic finite element method reflecting dynamic mesh characteristics is adopted. The real deformation and stress state of mesh tooth pair are simulated. $k_{i}$ can be obtained according to Eq.3. By wave filtering and other correlative numerical calculation, mesh stiffness coefficient of gear pair listed in Tab. 1 is obtained numerically as shown in Fig. 7.



Fig.8. Mesh error excitation

Table 1. Parameters of gear couples

\begin{tabular}{cccccc}
\hline & Num of teeth & Modification coefficient & Accuracy class & Modulus & Engaging angle $/^{\circ}$ \\
\hline The $1^{\text {st }}$ gear pair & 33 & -0.3 & $7 \mathrm{GJ}$ & 9 & 20 \\
& 26 & 0.3 & $7 \mathrm{GJ}$ & & \\
\hline The $2^{\text {nd }}$ gear pair & 37 & -0.3 & $7 \mathrm{GJ}$ & 9 & 20 \\
& 21 & 0.3 & $7 \mathrm{GJ}$ & & \\
\hline
\end{tabular}

The error excitation is actually the displacement dynamic excitation due to the difference between the real tooth profile surface and perfect surface. The mesh warp is frequently considered ${ }^{13}$. Mesh warp can be expressed by harmonic function. So sine function is adopted to simulate the error excitation ${ }^{5}$ as indicated in Fig. 8.

$$
e(t)=e_{0}+e_{r} \sin (2 \pi t / T+\Phi) .
$$

Where $e(t)$ is the mesh error; $e_{0}$ is the constant value of tooth error, $e_{0}=0$; $e_{r}$ is the amplitude of mesh error; $T$ is the tooth mesh periods. $\mathrm{e}_{\mathrm{r}}$ can be determined according to design accuracy class of gear pair. The effect of phase is not considered, $\Phi=0$.

\section{Bearing dynamic excitation}

Support bearings of the studied power gear transmission system are all ball bearings. It is assumed that balls roll in the certain orbit and bearing contact angle is the constant when it works. The contact pair of ball and outer ring is similar to that of ball and inner ring in geometry and kinematics. Contact parameters are similar. Hence, contact dynamic model is the same as that shown in Fig.9: 

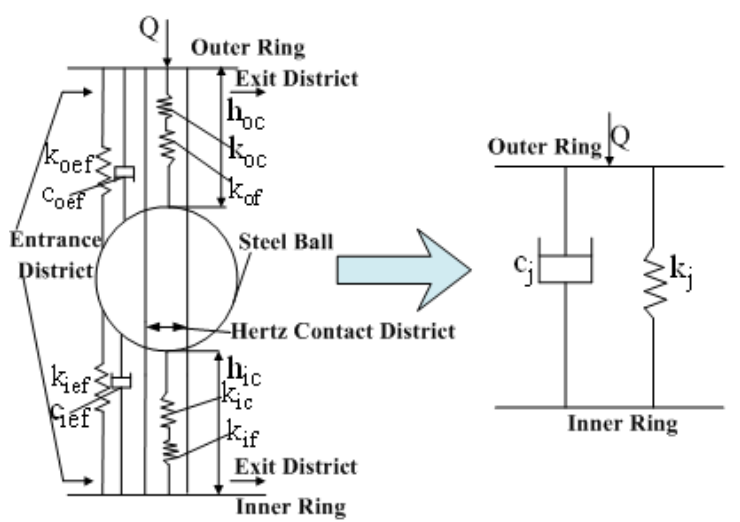

Fig.9. Dynamic model of ball bearing

The mathematic model is

$\left\{\begin{array}{l}k_{j}=\frac{\left(\left(1 / k_{i c}+1 / k_{i f}\right)^{-1}+k_{i e f}\right)\left(\left(1 / k_{o c}+1 / k_{o f}\right)^{-1}+k_{o f f}\right)}{\left(\left(1 / k_{i c}+1 / k_{i f}\right)^{-1}+k_{i e f}\right)+\left(\left(1 / k_{o c}+1 / k_{o f}\right)^{-1}+k_{o e f}\right)} . \\ c_{j}=c_{\text {oef }} c_{i e f} /\left(c_{o e f}+c_{i e f}\right)\end{array}\right.$

In entrance district, $k_{i e f}$ and $k_{\text {oef }}$ are the oil film stiffness coefficient of outer ring and inner ring respectively; $c_{\text {ief }}$ and $c_{\text {oef }}$ are the oil film damping coefficient of outer ring and inner ring respectively. In Hertz contact district, $k_{i c}$ and $k_{o c}$ are the contact stiffness coefficient between ball and inner and outer ring respectively; $k_{i f}$ and $k_{o f}$ are the oil film stiffness coefficient of outer and inner ring respectively.

The values of the coupled stiffness and dampness in different directions differ from each other under multi-direction work forces. The displacement vector inner ring relative to outer ring is

$$
u=\left\{x_{m}, y_{m}, z_{m}, \theta_{y m}, \theta_{z m}\right\}^{T} \text {. }
$$

where $x_{m}, y_{m}$ and $z_{m}$ are the displacements along the coordinate axial direction; $\theta_{y m}, \theta_{z m}$ are the angular displacements.

The basic correlation between load and displacement is mathematically described. Then stiffness and dampness matrixes are obtained based on the virtual working principle $^{14}$.

$$
k_{z}=k_{j} B \quad c_{z}=c_{j} B .
$$

Where

$$
B=\left[\begin{array}{ccccc}
z \sin ^{2} \alpha & 0 & 0 & 0 & 0 \\
0 & z \cos ^{2} \alpha / 2 & 0 & 0 & -z r \sin 2 \alpha / 4 \\
0 & 0 & z \cos ^{2} \alpha / 2 & z r \sin 2 \alpha / 4 & 0 \\
0 & 0 & z r \sin 2 \alpha / 4 & z r^{2} \sin ^{2} \alpha / 2 & 0 \\
0 & -z r \sin 2 \alpha / 4 & 0 & 0 & z r^{2} \sin ^{2} \alpha / 2
\end{array}\right] \text {; }
$$

$z$ is the number of balls; $\alpha$ is the contact angle; $r$ is the ball center circle radius.

As shown in Fig. 10, time-dependent curves of support stiffness coefficient and damping coefficient are calculated using MATLAB based on characteristic parameters of lubrication and material, bearing structure

\begin{tabular}{|c|c|c|c|c|c|c|c|c|}
\hline $\begin{array}{l}\text { Serial } \\
\text { number }\end{array}$ & $2 \mathrm{r} / \mathrm{mm}$ & $\begin{array}{l}\text { Ball } \\
\text { diameter/mm }\end{array}$ & $\alpha_{1}^{\circ}$ & $\begin{array}{l}\text { Roll orbit } \\
\text { curvature }\end{array}$ & $\begin{array}{l}\text { Flexible } \\
\text { modulus } \\
\text { / } \mathrm{N} / \mathrm{mn}^{2}\end{array}$ & $\begin{array}{l}\text { Poisson } \\
\text { ratio }\end{array}$ & $\begin{array}{l}\text { Oil } \\
\text { density } \\
/ \mathrm{Kg} / \mathrm{m}^{3}\end{array}$ & $\begin{array}{l}\text { Oil dynamic coefficient of } \\
\text { viscosity } / \mathrm{mm}^{2} / \mathrm{s}\end{array}$ \\
\hline Bearing1,3,6 & 110 & 20 & 35 & 0.531 & $2.1 \times 10^{5}$ & 0.33 & 860 & 80 \\
\hline Bearing2 & 125 & 22.5 & 35 & 0.5278 & $2.1 \times 10^{5}$ & 0.33 & 860 & 80 \\
\hline Bearing 5,8 & 140 & 25 & 35 & 0.525 & $2.1 \times 10^{5}$ & 0.33 & 860 & 80 \\
\hline Bearing 4 & 117.5 & 21.25 & 35 & 0.5294 & $2.1 \times 10^{5}$ & 0.33 & 860 & 80 \\
\hline Bearing 7,9 & 147.5 & 26.25 & 35 & 0.5428 & $2.1 \times 10^{5}$ & 0.33 & 860 & 80 \\
\hline
\end{tabular}
parameters listed in Tab.2 and dynamic load.

Table 2 Parameters of each bearing 


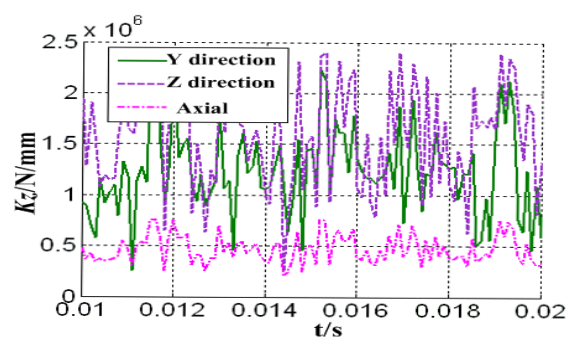

a) Bearing stiffness

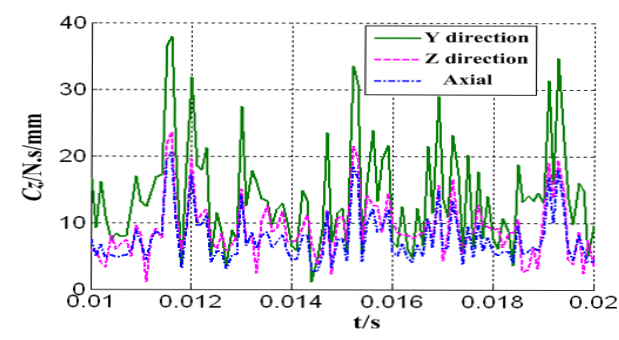

b) Bearing dampness

Fig.10. Stiffness coefficient and damp curve of the 4th bearing

Fig. 10 shows values of stiffness and dampness are time-dependent due to the change of interactional forces between ball and its orbits, and mutative tendency of stiffness and dampness in different radial directions are similar though small numerical value error exists.

\section{Simulation analysis}

\subsection{System model construction}

Analysis model of power gear train system is developed based on dynamic substructure method. Gear train system can decompose transmission shafts, gear pair and rolling bearing. System dynamic model at certain gear ratio is shown in Fig. 11.

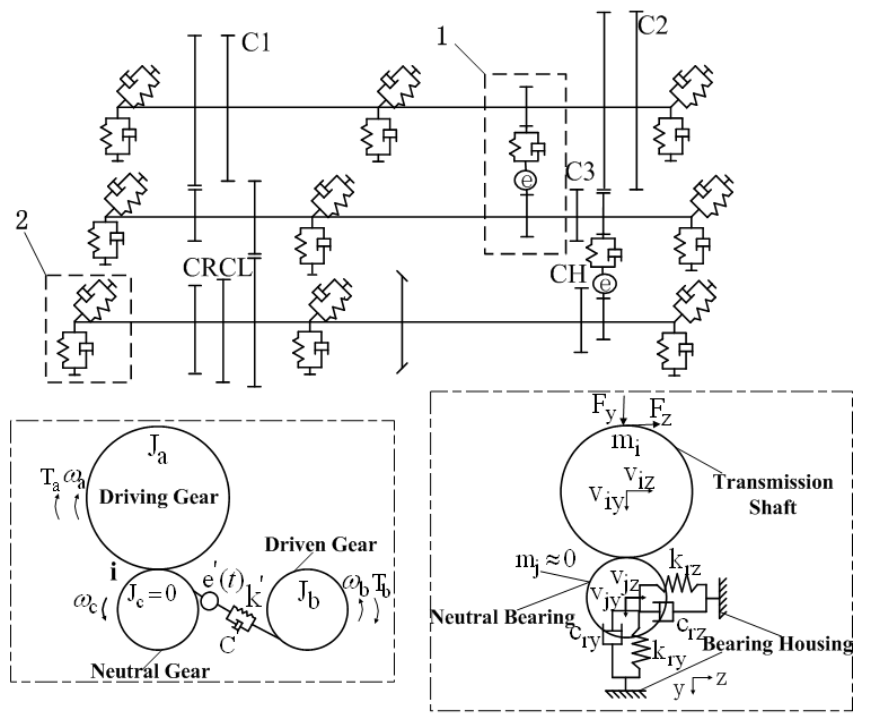

11-1 Modified Gear Pair 11-2 Dynamic Model of Shaft-Bearing System

Fig.11. Dynamic model of gear train system

Transmission shafts are considered as flexible shafts. Mode analysis is based on the finite element model of shafts. The shaft flexibility which can be described with its mode characteristics can be brought into dynamics equation of gear system by coordinate transformation.

Gear joint in ADAMS can't simulate dynamic mesh state. The new model of gear pair should be modified. A neutral gear without mass is built. Gear joint is created between the neutral gear and driving gear. Torsional spring connecting neutral gear and driven gear can portray dynamics behaviour caused by inner excitations. Corrected dynamic model of gear pair is built as shown in Fig. 11-1. The corrected mathematic model is given by

$$
\left\{\begin{array}{l}
T_{a}-\frac{\left[K^{\prime} e^{\prime}(t)+K^{\prime} \int\left(\omega_{c}-\omega_{b}\right) d t+C^{\prime}\left(\omega_{c}-\omega_{b}\right)\right]}{i}=J_{a} \dot{\omega}_{a} \\
{\left[K^{\prime} e^{\prime}(t)+K^{\prime} \int\left(\omega_{b}-\omega_{c}\right) d t+C^{\prime}\left(\omega_{b}-\omega_{c}\right)\right]-T_{b}=J_{b} \dot{\omega}_{b}}
\end{array}\right.
$$

where $T_{a}$ is the driving torque; $T_{b}$ is the driven torque; $\theta_{a}$ is the rotational angle of driving gear; $\theta_{b}$ is the rotational angle of neutral gear; $\theta_{c}$ is the rotational angle of driven gear; $K$ is the equivalent torsional stiffness coefficient, $K^{\prime}=K R_{b}^{2} ; C^{\prime}$ is the equivalent torsional damping coefficient, $C^{\prime}=C R_{b}^{2} ; e^{\prime}(t)$ is the equivalent mesh error, $e^{\prime}(t)=e(t) / R_{b} ; R_{b}$ is the basic circle radius of driven gear; $i$ is the gear ratio.

Neutral parts without mass are created in which there is a bearing. Planer joint is created between neutral parts and bearing seat, which can ensure bearing kinematics movement. Bearing dynamic characteristic is simulated 
by creating bushing between transmission shaft and bearing seat. Therefore, as shown in Fig. 11-2, dynamic model of shaft-bearing system is constructed. The mathematic model is

$\left\{\begin{array}{c}F_{y}=m_{i} \dot{v}_{i y}+c_{r y} v_{i y}+k_{r y} \int v_{i y} d t \\ F_{z}=m_{i} \dot{v}_{i z}+c_{r z} v_{i z}+k_{r z} \int v_{i z} d t\end{array} \quad\left\{\begin{array}{c}v_{i y}=v_{j y} \\ v_{i z}=v_{j z}\end{array}\right.\right.$.

where $F_{y}$ and $F_{z}$ are the support forces in $\mathrm{y}$ and $\mathrm{z}$

direction respectively; $m_{i}$ is the mass of transmission

shafts; $v_{i y}$ and $v_{i z}$ are the relative velocity of transmission shaft versus bearing housing in $\mathrm{y}$ and $\mathrm{z}$ direction respectively; $v_{j y}$ and $v_{j z}$ are the relative velocity of neutral part versus bearing housing in $\mathrm{y}$ and $\mathrm{z}$ direction respectively; $c_{r y}$ and $c_{r z}$ are the damping coefficients in $\mathrm{y}$ and $\mathrm{z}$ direction respectively; $k_{r y}$ and $k_{r z}$ are the stiffness coefficients in $\mathrm{y}$ and $\mathrm{z}$ direction respectively.

Constraint function is built under partial constraints based on aforementioned three dynamic sub-structures. The kinematics parameters and time-dependent stress and strain of any points in shafts can be numerically calculated. Fig. 12 shows the prototype of power gear transmission system.

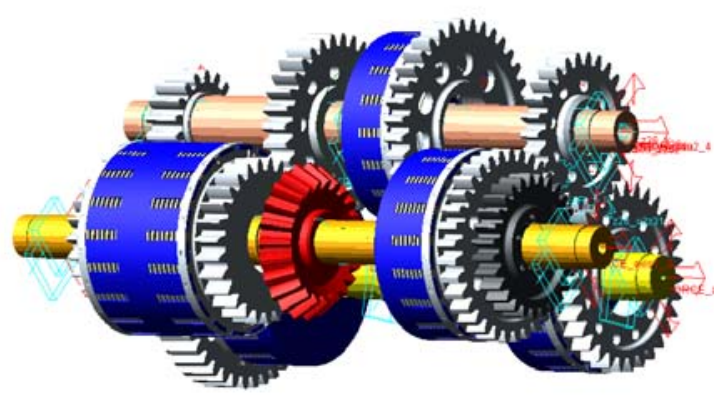

Fig.12. System virtual prototype

\subsection{Results}

Engine fluctuant excitation is equivalently taken to the input gear. Road rolling resistance coefficient is 0.05 . Equivalent load is taken to the both sides of the 3rd shaft. Visualized simulation of system acceleration can be realized by creating multi-excitation. System works at fixed gear ratio steadily eventually. Dynamic characteristics of its steady state are discussed.

\subsubsection{Dynamic response}

Gear mesh forces and displacement responses of shafts in the center mesh gear are analyzed to describe the system dynamic characteristics.

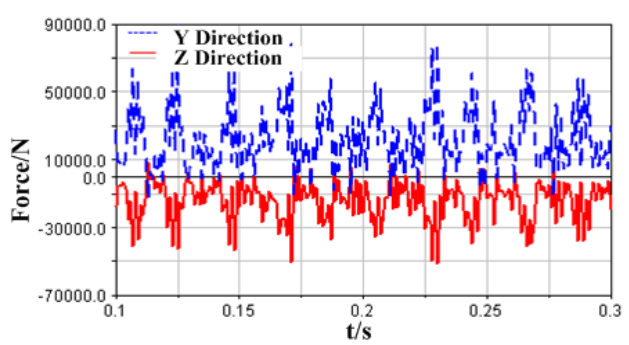

Fig.13. Mesh force of the first couple gears

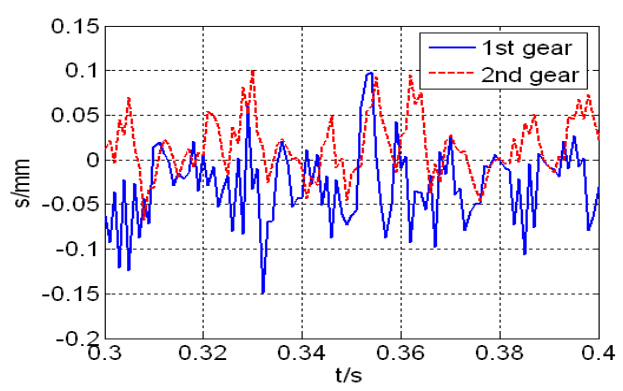

Fig.14. Displacement response of mesh gears center points

As indicated in Fig. 13, mesh forces fluctuate periodically. Variation tendency is in agreement with gear kinetic trait. The period of mesh force fluctuation is equal to the mesh time of one couple of gear teeth in a cycle. It can be validated by the theory that the root of mesh force undulation is the value of coincidence degree.

Displacement response (Fig. 14) moves with time in waves. Response absolute values of different shafts in mesh gears center points are close to each other. Phase difference is $180^{\circ}$. 


\subsubsection{Output angular velocities in various excitations}

Output angular velocity is the crucial factor to reflect the dynamic characteristics. So output angular velocity contrast in various excitations is analyzed.



a) Effect of the bearing

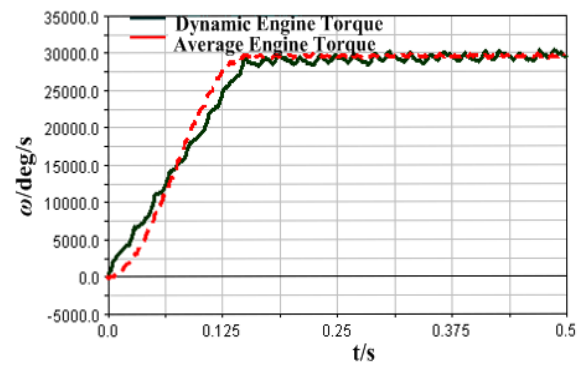

b) Effect of the engine

Fig.15. Effect on output angular velocity by various excitations

Fig. 15 shows that output angular velocity fluctuates near the theoretic value because of the effect of multi-excitations when system works at fixed gear ratio. Its maximum error is $4.61 \%$ which is accredited. Support flexibility has little impact on the output angular velocity. There is the certain correlation between engine dynamic torque and output angular velocity. The curve is smoother when engine average torque is adopted.

\subsubsection{Support forces in different excitation}

Support forces offer the boundaries condition to study on gearbox and transmission shafts dynamic traits, which is investigated.

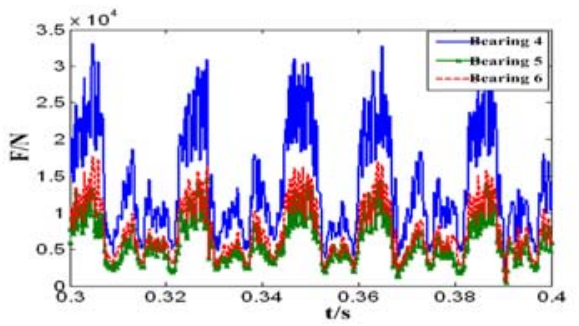

a) With the consideration of bearing flexibility

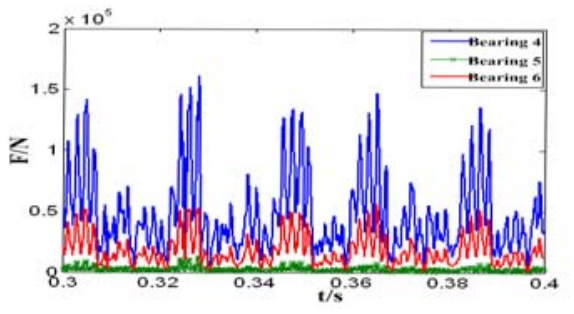

b) without the consideration of bearing flexibility

Fig.16. Effect on support forces by the bearing flexibility

Fig. 16 shows that support-force time history is similar in different support condition, though amplitudes of them differ with each other significantly. Support flexibility as the cushion against impact reduces the value of support force. It cycles in time-domain more stably. System impact decreases apparently. In the different support condition, the allocation proportion of each support force in one transmission shaft is also different. The value of the 4th bearing and the 6th bearing support forces are much greater than that of the 5 th bearing without the consideration of bearing flexibility. The amplitude of each support force is close to each other in consideration of bearing flexibility. Therefore, the latter takes the interaction of each bearing into account. Simulation results with bearing flexibility are closer to real working conditions.

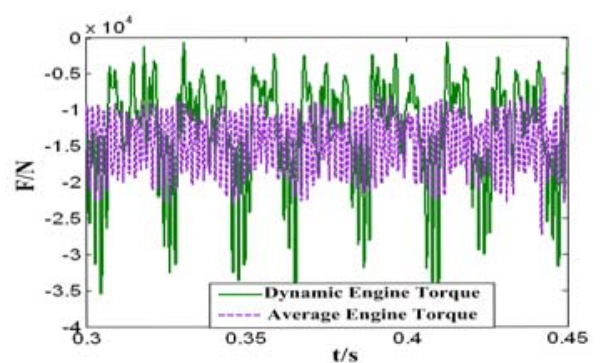

a) Time domain

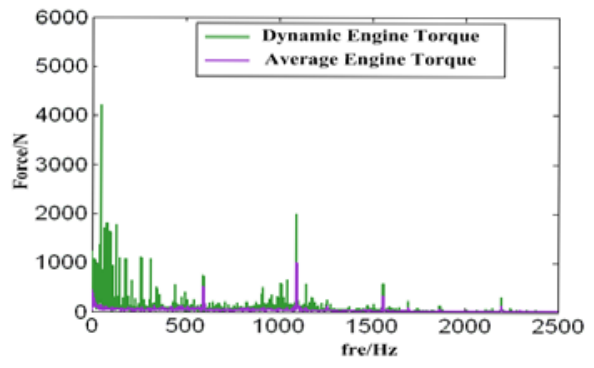

b) Frequency domain

Fig.17. Effect of engine excitation on support force (Y) 
Fig. 17 shows the effect of engine dynamic excitation on support force. In time-domain, support force cycles evenly and the value of peak force is close to each other when adopting average engine torque; support force fluctuates significantly because of time-dependent factor in engine excitation when engine dynamic excitation is included. Due to the effect of gear inner excitation, in frequency-domain the peak points of force amplitude occur at about $1100 \mathrm{~Hz}$ and $1565.5 \mathrm{~Hz}$, which are the mesh basic frequencies of two couples of gear pair. However, other peak points focusing on the lower frequency district below $500 \mathrm{~Hz}$, which are the engine impulse frequencies, appear when engine dynamic excitation is taken into account. Hence, engine torsional excitation affects the system characteristics more greatly than other source excitations.

\section{Conclusions}

Multi-source excitations are simulated numerically. Prototype of rigid and flexible coupled power gear train system is created in consideration of multi-source excitations. The feasibility of acquiring the time spectrum of dynamic response of any position in transmission shafts is validated. Simulation results indicates time-dependent mesh force is in agreement with the theoretical dynamic mesh force caused by variable mesh stiffness coefficient; The simulation in consideration of bearing flexibility takes the interaction of each bearing in one shaft into account and simulation results are closer to the real working condition; Engine excitation focuses on the lower frequency district and affects the system characteristic significantly.

\section{Acknowledgements}

This work was partially supported by Natural Nature Science Foundation of China (50905018). The authors would like to express gratitude to its financial support.

\section{References}

1. A.Kaharman, Nonlinear dynamics of a spur gear pair, Journal of Sound and Vibration. 103(2)(1990), 447-459.

2. T.Tsuta, Excitation force analysis of helical gear-pair with tolerance in their tooth shape and pitch mounted on flexible shaft, MPT91 JSME International Conference on
Motion and Power Transmission, Hiroshima, Japan. 23-26(1991), 72-77.

3. R.G.Parker ,S.M.Vijayakar and T.Imajo, Non-linear dynamic response of a spur gear pair: modeling and experimental comparisons, Journal of Sound and Vibration. 237(3)(2000), 435-455.

4. L.Vedmar and A.Andersson, A method to determine dynamic loads on spur gear teeth and on bearings. Journal of Sound and Vibration, 37(2002), 1065-1084.

5. Runfang Li, Zeguang Tao, Tengjiao Lin and Qian Tang, Numerical simulation for inner dynamic excitation of gearing, Mechanical Transmission. 25(2)(2001), 1-3.

6. Tengjiao Lin, Runfang Li, Chengyun Yang and Huajiang Hang, Numerical simulation for inner dynamic excitation and system vibration response of speed increasing gearbox, Transaction of Chinese Society for Agricultural Machinery. 33(6)(2002), 20-22.

7.Chengyun Yang, Study on coupled vibration response and anti-impact property of gear transmissions system, $P H D$ Thesis at Chongqing University, Chongqing, China, 2006.

8. Abdelhamid Bouchachia, Kyandoghere Kyamakya, Computational Intelligence Applied to Nonlinear Dynamics and Synchronization, International Journal of Computational Intelligence Systems. Vol.2, No. 2 (June, 2009), 99-103.

9. Qingquan Hong and Ying Cheng, Dynamic Simulation of Multistage Gear Train System in ADAMS, Transactions of Beijing Institute of Technology. 23(6)(2003), 690-693.

10. Zhirui Qian, Xiaoyan Huang, Mingzhe $\mathrm{Li}$ and dongping Li, Spinning Transmission System Dynamics Simulation, China Mechanical Engineering. 17(3)(2006), 241-244.

11. Yan Wang, Changzhi Jia, Xiaoping Han and Mingshan Luo. Virtual Prototyping and Simulation on Multi Rotor-Bearing System, Journal of Ordnance Engineering College. 19(3)(2007), 50-53.

12. Tingqiang Yao, Yilin Chi, Yayu Huang and Yang Tan, Contact Dynamics Simulation of Rigid and Flexible Coupled Spindle System, Mechanical Science and Technology for Aerospace Engineering. 26(11)(2007), 1507-1510.

13. Runfang Li and Jianjun Wang, Dynamics of Gear System, Science Press, Beijing, China, 1997.

14. Lianchun Zhao, Research on Vibration of Ball Bearings, PHD Thesis at Zhejiang University, Zhejiang, China, 2003. 